Research Article

iMedPub Journals

www.imedpub.com

\section{Accuracy of Percutaneous Pedicle Screw Placement in Thoracolumbar Spines: A Comparison Study Between Experienced Spine Surgeons and Residents}

\section{Abstract}

Purpose: The aim of this study was to investigate the difference in the accuracy of percutaneous pedicle screws (PPSs) insertion between spine surgery specialists and residents in cases of thoracolumbar surgery.

Methods: We evaluated the insertion accuracy of 355 PPSs (left 178, right 177) used in 38 patients with thoracolumbar diseases surgically treated in our hospital between April 2009 and March 2016. A spine surgery specialist served as the main operator in 20 cases and a resident served as the main operator in 18 cases with a spine surgery specialist as the assistant. Using CT horizontal section images obtained within 2 weeks after surgery, the site of PPS insertion was evaluated according to four grades (Excellent, Good, Fair, Poor) in terms of six locations (la, $\mathrm{Ib}, \mathrm{Ila}, \mathrm{Il} \mathrm{b}, \mathrm{IIla}, \mathrm{IIlb})$, mechanical fixation, and risk of occurrence of complications.

Results: Among all 355 PPSs, la (Excellent) was achieved in 343 (96.6\%). Twelve PPS insertions were classified as other grades, showing a deviation rate of $3.4 \%$. All 12 of these insertions were rated as $\mathrm{lb}$ (Good), and no other grades were identified. Spine surgery specialists performed 3 PPS insertions rated as Ib (Good), whereas residents performed 9 insertions rated as Ib (Good). There was no statistically significant difference between specialists and residents $(P=0.083)$. All 12 insertions rated as other than la (Excellent) were insertions on the right side, but there was no consistent trend in terms of patient age or gender, disease, presence/absence of osteoporosis, spinal level, PPS model, or the location of the fluoroscopic system.

Conclusion: PPS fixation is a safe procedure that allows for very accurate screw insertion by operators with limited clinical experience.

Keywords: Percutaneous pedicle screw; Spinal fixation; Accuracy; Clinical experience

\author{
Funayama T* and \\ Tsukanishi T \\ Department of Orthopaedic Surgery, \\ Kenpoku Medical Centre, Takahagi Kyodo \\ Hospital, Japan
}

Corresponding author: Toru Funayama

Đ funatoru3@gmail.com

Department of Orthopaedic Surgery, Kenpoku Medical Centre, Takahagi Kyodo Hospital, 1006-9 Agehocho, Kamitetsuna, Takahagi, Ibaraki 3180004, Japan.

Tel: $+81-293-231122$

Fax: $+81-293-241063$

Citation: Funayama T, Tsukanishi T (2017) Accuracy of Percutaneous Pedicle Screw Placement in Thoracolumbar Spines: A Comparison Study Between Experienced Spine Surgeons and Residents. Spine Res. Vol.4 No.1:1

Received: December 29, 2017; Accepted: January 25, 2018; Published: January 29, 2018

\section{Introduction}

Thoracolumbar pedicle screws have been widely used for various spinal diseases. Accurately inserted pedicle screws are useful for spinal fusion, contributing to the stability of the spine. However, deviation of pedicle screws outside the vertebral body jeopardizes spinal stability and may cause vascular or visceral injury. If the screw is deviated into the vertebral canal, there is a risk of nerve injury. Therefore, sufficient clinical experience and adequate technical skills are required to achieve safe and accurate insertion of pedicle screws when the conventional freehand method is used [1,2].

Recent years have seen the advent of percutaneous pedicle screws (PPSs) and their application to various minimally invasive spinal operations [3-5]. We have been using PPSs since April 2009. In our hospital, the surgical team for a spinal operation often includes an inexperienced orthopaedic surgery resident 
because of the limited staff of orthopaedic surgeons. However, there has been no major difficulty in PPS placement. Our search of the English literature yielded one article, examining lumbar and sacral (L2-S1) vertebrae, that discussed the variation in PPS insertion accuracy according to clinical experience of the operator [6], but no reports evaluating lower thoracic or upper lumbar vertebrae, where the pedicle diameter is smaller were found. It is important to know the accuracy of PPS insertion in a less-experienced surgeons for the safe surgery. In this study, we investigated the difference in the accuracy of PPS insertion between spine surgery specialists and residents in cases of thoracolumbar surgery. This study was one of the first reports on this topic. We hypothesized that even less-experienced surgeons could perform safe pedicle screw insertion as well-experienced surgeons using percutaneous insertion technique.

\section{Materials and Methods}

The study protocol was approved by the institutional review board at Kenpoku Medical Center Takahagi Kyodo Hospital (approval number TKHIRB-2017-04-001). All patients provided informed consent for this study.

We performed a cross-sectional study of 355 PPSs (left 178, right 177) used in consecutive 33 patients with thoracolumbar diseases surgically treated with PPS in our hospital between April 2009 and March 2016. All cases were treated by a surgical team consisted of a spine surgery specialist and resident. Patients were excluded from the study if they were treated by only spine surgery specialists. The thoracolumbar diseases included 19 cases of traumatic vertebral fracture (including 2 cases of vertebral fracture associated with diffuse idiopathic skeletal hyperostosis), 17 cases of delayed union or pseudarthrosis after osteoporotic vertebral fracture, 1 case of lumbar spondylolisthesis, and 1 case of spinal caries. The pedicle screw system, chosen by the attending doctor arbitrarily according to the individual case, was ILLICO (Alphatec Spine, Carlsbad, California, USA) in 18 cases, MANTIS (Stryker, Kalamazoo, Missouri, USA) in 7 cases, SEXTANT (Medtronic Sofamor Danek, Memphis, Tennessee, USA) in 5 cases, ES2 (Stryker) in 3 cases, PANGEA (Depuy Synthes, Raynham, Massachusetts, USA) in 2 cases, IBIS (MDM, Tokyo, Japan) in 2 cases, and VOYAGER (Medtronic) in 1 case.

In principle, the operation was performed by 1 of 4 staff specialists in spine surgery with orthopaedic clinical experience of at least 10 years, paired with 1 of 8 residents, with orthopaedic clinical experience of 2-4 years. The main operator was in charge of PPS insertion on the left side, whereas the assistant was in charge of PPS insertion on the right side. A spine surgery specialist served as the main operator in 20 cases and as the assistant in 18 cases. A resident served as the main operator in 18 cases and as the assistant in 20 cases. A fluoroscopic X-ray system (C-arm) was set on the right side of the patient in the initial 14 cases, and on the left side in the subsequent 9 cases. Two fluoroscopic X-ray systems were used in the last 15 cases to reduce the fluoroscopy time and the risk of contamination of the operative field. PPSs were inserted either via a small incision of the skin and fascia or via a posterior midline incision of the skin combined with small incision of the fascia, according to the individual case. The screw diameter that matched the pedicle diameter was chosen based on preoperative computed tomography (CT) horizontal section images. After confirming that the tip of the pack needle was inserted into the PPS insertion point, i.e., the craniolateral side of the pedicle, in the antero-postero fluoroscopic view, the pack needle was moved forward manually in a prudent manner. When the pack needle tip was judged to be in the optimal site by confirming that the tip reached the posterior border of the vertebral body in the lateral view but remained in the inside of the pedicle in the antero-postero view [4], it was advanced continuously to the center of the vertebral body. Then, the guide wire was set there, and a PPS was inserted.

Using CT horizontal section images obtained within 2 weeks after surgery, the site of PPS insertion was evaluated according to four grades (Excellent, Good, Fair, Poor) in terms of six locations (la, Ib, Ila, IIb, IIla, IIIb), mechanical fixation, and risk of occurrence of complications, based on reports by Heintel et al. [5] and Zdichavsky et al. [7]. More specifically, insertion status of each screw was determined as la (excellent) when the screw was inserted into the vertebral body through the pedicle, Ib (Good) when the screw perforated the lateral bone cortex of the pedicle and was inserted into the vertebral body, Ila (Fair) when the screw passed through the pedicle but deviated outside of the vertebral body, Ilb (Fair) when less than half of the screw diameter deviated inside the spinal canal, Illa (Poor) when the screw perforated the lateral bone cortex of the pedicle and further deviated outside of the vertebral body, and IIIb (Poor) when half or more of the screw diameter deviated inside the spinal canal. A single-blind CT evaluation of the location of the inserted PPS was performed by an orthopedic specialist, independent of this study.

The accuracy of PPS insertions by spine surgery specialists (176 PPSs) was compared with that by residents (179 PPSs), using the chi-squared test (significance level $P<0.05$ ), and PPS insertions with any rate other than la (excellent) were analyzed in detail. All analyses were performed with Statcel2 (OMS Inc, Saitama, Japan).

\section{Results}

Among the 38 patients, the spine levels at which PPSs were inserted were T9-L5. The number of PPSs in relation to the spine level was as follows: 8 screws at T9, 34 at T10, 60 at T11, 65 at T12, 64 at L1, 60 at L2, 40 at L3, 18 at L4, and 6 at L5. Among all 355 PPSs, la (Excellent) was achieved in 343 (96.6\%). Twelve PPS insertions were classified as other grades, showing a deviation rate of $3.4 \%$ (Figure 1). All 12 of these insertions were rated as $\mathrm{lb}$ (Good), and no other grades were identified. Spine surgery specialists performed 3 PPS insertions rated as Ib (Good), whereas residents performed 9 insertions rated as Ib (Good) (Table 1). There was no statistically significant difference between specialists and residents $(P=0.083)$. All 12 insertions rated as other than la (Excellent) were insertions on the right side, but there was no consistent trend in terms of patient age or gender, disease, presence/absence of osteoporosis, spinal level, PPS model, or the location of the fluoroscopic system (Table 2) and (Table 3). Representative la and Ib cases respectively (Figures 2 and 3$)$. 


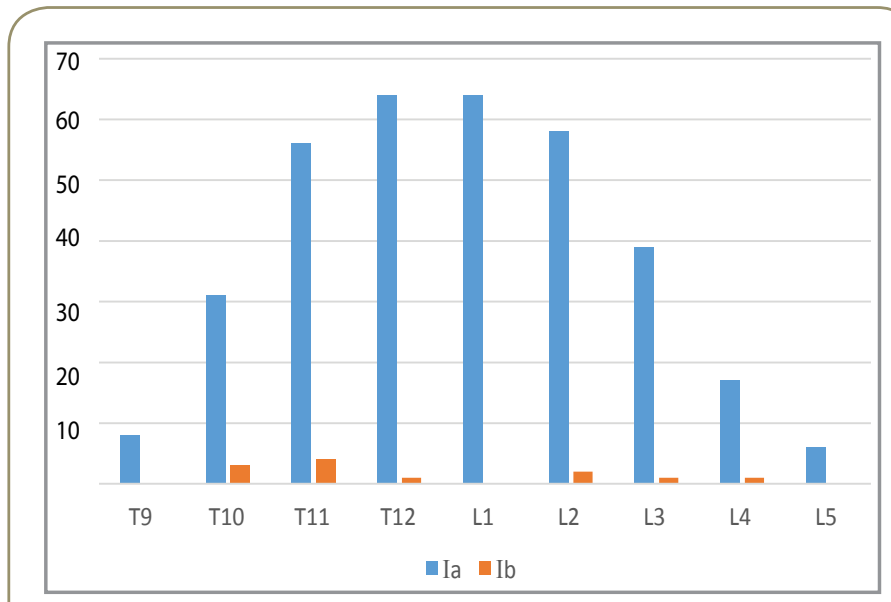

Figure 1 The spine levels at which PPSs were inserted. The number of PPSs in relation to the spine level was as follows: 8 screws at T9, 34 at T10, 60 at T11, 65 at T12, 64 at L1, 60 at L2, 40 at L3, 18 at L4, and 6 at L5. Among all 355 PPSs, la

Table 1 The evaluation of PPSs accuracy among spine surgery specialists and residents.

\begin{tabular}{|c|c|c|c|}
\hline Title & Ia & Ib & Total \\
\hline SSS & 173 & 3 & 176 \\
\hline RSD & 170 & 9 & 179 \\
\hline Total & 343 & 12 & 355 \\
\hline
\end{tabular}

Note: $-p=0.083$

SSS: Spine Surgery Specialist; RSD: Resident; la (excellent): The screw was inserted into the vertebral body through the pedicle; Ib (good): The screw perforated the lateral bone cortex of the pedicle and was inserted into the vertebral body

Table 2 Summary of cases with non-"Ia" screws.

\begin{tabular}{|c|c|c|c|c|}
\hline Case & \multicolumn{2}{|c}{ Sex } & Disease & Osteoporosis \\
\hline 1 & 83 & F & OVF pseudarthrosis & Yes \\
\hline 2 & 57 & M & Vertebral fracture & No \\
\hline 3 & 79 & F & OVF pseudarthrosis & Yes \\
\hline 4 & 79 & F & OVF pseudarthrosis & Yes \\
\hline 5 & 74 & F & OVF pseudarthrosis & Yes \\
\hline 6 & 72 & F & OVF pseudarthrosis & Yes \\
\hline 7 & 46 & M & Vertebral fracture & No \\
\hline 8 & 78 & F & OVF pseudarthrosis & Yes \\
\hline 9 & 78 & F & OVF pseudarthrosis & Yes \\
\hline 10 & 66 & M & Vertebral fracture & No \\
\hline 11 & 82 & M & Vertebral fracture & No \\
\hline 12 & 77 & F & Vertebral fracture & Yes \\
\hline
\end{tabular}

Table 3 Summary of cases with non-"Ia" screws.

\begin{tabular}{|c|c|c|c|c|c|}
\hline Case & $\begin{array}{c}\text { Spinal level and } \\
\text { position o }\end{array}$ & Evaluation & PPS model & $\begin{array}{c}\text { Position of } \\
\text { C-arm }\end{array}$ & SSS/RSD \\
\hline 1 & L2 right & Ib & Illico & Right & SSS \\
\hline 2 & T10 right & Ib & Pangea & Right & RSD \\
\hline 3 & T11 right & Ib & Illico & Right & RSD \\
\hline 4 & L3 right & Ib & Illico & Right & RSD \\
\hline 5 & T11 right & Ib & Illico & Right & RSD \\
\hline 6 & T10 right & Ib & Illico & Left & RSD \\
\hline 7 & L4 right & Ib & Mantis & Left & SSS \\
\hline 8 & T11 right & Ib & Illico & Left & RSD \\
\hline 9 & L2 right & Ib & Illico & Left & RSD \\
\hline 10 & T10 right & Ib & Mantis & Left & SSS \\
\hline 11 & T11 right & Ib & Illico & Double & RSD \\
\hline 12 & T12 right & Ib & ES2 & Double & RSD \\
\hline
\end{tabular}

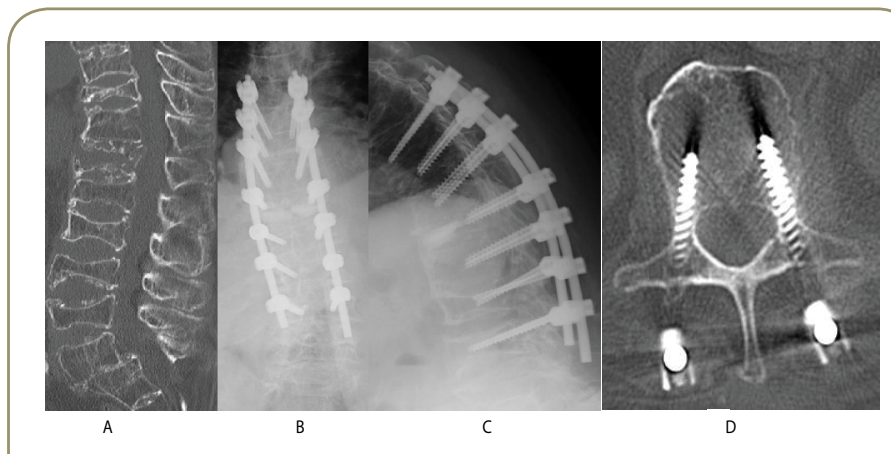

Figure 2 An 86-year-old female, A: T12 vertebral fracture associated with DISH (diffuse idiopathic skeletal hyperostosis). B and C: PPS were inserted from T9 to L3. D: All screws were evaluated as la (excellent) which the screw was inserted into the vertebral body through the pedicle.

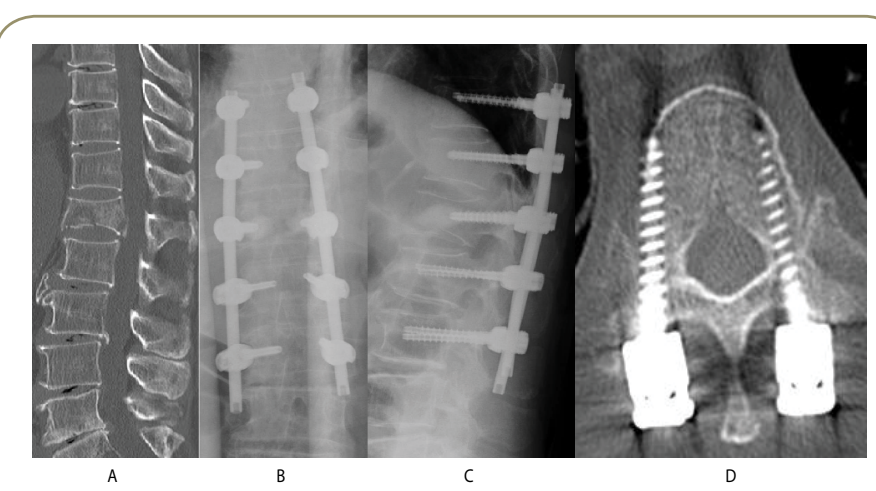

Figure 3 An 83-year-old male, A: L1 vertebral fracture. B and C: PPS were inserted from T11 to L3. D: The screw of right T11 was evaluated as Ib (good), which the screw perforated the lateral bone cortex of the pedicle and was inserted into the vertebral body. 


\section{Discussion}

This study showed that the deviation rate was very low at $3.4 \%$. This indicates that PPSs can be inserted with very high accuracy and safety by the insertion method we adopted. The accuracy of PPS insertion has occasionally been reported, and recent reports have shown that the frequency of deviation from the optimal insertion site of the screw is $2 \%$ to $23 \%[4,5,8,9]$. To insert PPSs safely, Raley et al. [4] state that it is important to confirm that the tip of the guide pin or the hollow probe remain inside the pedicle in the antero-postero view when it reaches the posterior border in the lateral view. We also regard this point as most important. It is necessary to accurately assess the location of the pack needle inside the pedicle or the vertebral body in a 3-dimensional manner by using 2-dimensional fluoroscopic images obtained during surgery. Our method is no different from the conventional method, in that there is always the risk of deviation outside the vertebral body or inside the spinal canal. In addition, both our method and the conventional method require that the operator have full knowledge of the anatomy of each pedicle prior to surgery.

This study revealed no statistically significant difference in the accuracy of PPS insertion between spine surgery specialists and residents, showing that accurate PPS insertion is feasible regardless of the number of years of clinical experience if the operation is performed under the direction of a senior surgeon. The reason for this is probably that the information on the positions of pack needles and screws can always be shared between the spine surgery specialist and the resident because all serial procedures are performed under fluoroscopy. We believe that, for orthopaedic surgeons who do not have enough experience, the PPS is a device that allows very safe insertion, providing greater visual information than the conventional pedicle screws that are inserted free-hand.

In this study, PPS insertions on the left side were all rated as la, and insertions with any rate other than la were all on the right side. Lee et al. [10] reported that deviation of the screw was more likely to occur on the right side when the operator standing

\section{References}

1 Amato V, Giannachi L, Irace C, Corona C (2010) Accuracy of pedicle screw placement in the lumbosacral spine using conventional technique: computed tomography postoperative assessment in 102 consecutive patients. J Neurosurg Spine 12: 306-313.

2 Gang C, Haibo L, Fancai L, Weishan C, Qixin C (2012) Learning curve of thoracic pedicle screw placement using the free-hand technique in scoliosis: how many screws needed for an apprentice? Eur Spine J 21: 1151-1156.

3 Ni WF, Huang YX, Chi YL, Xu HZ, Lin Y, et al. (2010) Percutaneous pedicle screw fixation for neurologic intact thoracolumbar burst fractures. J Spinal Disord Tech 23: 530-537.

4 Raley DA, Mobbs RJ (2012) Retrospective computed tomography scan analysis of percutaneously inserted pedicle screws for posterior on the left side of the patient also inserted the pedicle screw on the right side by the conventional free-hand method, although the reason remains undetermined. In the present study, the main operator (the attending doctor of the patient) was always in charge of left-sided screws, and the assistant was always in charge of right-sided screws. Because there was no consistent trend with regard to patient age or gender, disease, presence/ absence of osteoporosis, spinal level, screw model, or location of the fluoroscopic system, the reason for the difference in PPS insertion between the left and right side remains unclear. It is desirable to use more caution when inserting PPSs in the future, taking into account the possibility of screw deviation to the lateral side of the pedicle during the insertion procedure.

As safety and accuracy of PPS insertion is pursued further, more fluoroscopy time is required. In this study the fluoroscopy time was not measured between spine surgery specialist and resident. Residents may need more fluoroscopic time for safe PPS insertion. This point was one important limitation in this study. Although it has been reported that the operator's exposure to radiation during PPS insertion is within the safe range [11], it is necessary in the future to aim for reduced radiation exposure to both the patient and the operator by reducing the fluoroscopy time to the maximum possible extent while maintaining the accuracy of screw insertion.

\section{Conclusion}

Percutaneous thoracolumbar pedicle screw fixation was associated with a very low deviation rate of $3.4 \%$, irrespective of the operator's clinical experience. PPS fixation is a safe procedure that allows for very accurate screw insertion by operators with limited clinical experience when performed under the direction of a spine surgery specialist.

\section{Acknowledgement}

The authors express their sincere gratitude to Dr. Hiroshi Wada (Board certified surgeon by the Japanese Orthopaedic Association), who performed CT evaluation in this study.

transpedicular stabilization of the thoracic and lumbar spine. Spine 37: 1092-1100.

5 Heintel TM, Berglehner A, Meffert R (2013) Accuracy of percutaneous pedicle screws for thoracic and lumbar spine fractures: a prospective trial. Eur Spine J 22: 495-502.

6 Nakahara M, Yasuhara T, Inoue T, Takahashi Y, Kumamoto S, et al. (2016) Accuracy of percutaneous pedicle screw insertion technique with conventional dual fluoroscopy units and a retrospective comparative study based on surgeon experience. Global Spine J 6: 322-328.

7 Zdichavsky M, Blauth M, Knop C (2004) Accuracy of pedicle screw placement in thoracic spine fractures Part I: inter- and intra-observer reliability of the scoring system. Eur J Trauma 30: 234-240.

8 Ravi B, Zahrai A, Rampersaud R (2010) Clinical accuracy of computer- 
assisted two-dimensional fluoroscopy for the percutaneous placement of lumbosacral pedicle screws. Spine 36: 84-91.

9 Oh HS, Kim JS, Lee SH, Liu WC, Hong SWE (2013) Comparison between the accuracy of percutaneous and open pedicle screw fixations in lumbosacral fusion. Spine J 13: 1751-1757.

10 Lee CH, Hyun SJ, Kim YJ, Kim KJ, Jahng TA, et al. (2014) Accuracy of free hand pedicle screw installation in the thoracic and lumbar spine by a young surgeon: an analysis of the first consecutive 306 screws using computed tomography. Asian Spine J 8: 237-243.

11 Mroz TE, Abdullah KG, Steinmetz MP, Klineberg EO, Lieberman IH (2011) Radiation exposure to the surgeon during percutaneous pedicle screw placement. J Spinal Disord Tech 24: 264-267. 